\title{
Clinical Trials for Patients with Fibromyalgia Syndrome
}

\section{Kim Lawson*}

Biomedical Research Centre, Sheffield Hallam University, Faculty of Health and Wellbeing, City Campus, Sheffield, S1 1WB, UK

Fibromyalgia syndrome (FM) is a chronic condition that is dominated by widespread pain, but presents with a complex of symptoms that include fatigue, dysfunctional sleep and cognitive disruption [1]. Patients have been diagnosed in most countries across the world with a prevalence of FM estimated to be $2-4 \%$ of the population. Progress has recently been made in understanding aspects of the pathophysiology and identification of effective treatments of FM [1,2], although there is still a reliance on the subjectivity of symptom profiles as identified by the American College of Rheumatology (ACR) criteria for diagnosis [3]. The pathophysiology of FM has been suggested to involve dysfunction of the central nervous system pain modulatory systems leading to central sensitization, dysfunction of the neuroendocrine system and dysautonomia [1,2]. Neuroimaging has indicated abnormalities in regional cerebral blood flow, in the white and gray matter and the brain metabolites (glutamate, glutamine, creatine and $\mathrm{N}$-acetyl-aspartate) in individuals with FM [4]. The complexity of symptoms that results is then associated with a heightened generalized sensitization to sensory input.

The current therapeutic approach to improve health status in FM is a multi-modal rehabilitation model, integrating exercise, behavioural/management programs and pharmacological treatment; however, a universally accepted treatment algorithm is often lacking $[1,5]$. A diversity of physical and psychological characteristics of the symptoms suggestive of a heterogeneous population of patients further complicates the treatment approach [6]. In addition, the relative lack of reliability of the application of the ACR criteria, which has aspects that overlap with many symptoms of other health conditions (eg chronic fatigue syndrome, ME, myofascial pain), for the diagnosis of FM may miss patients [7].

Although a variety of treatment modalities have demonstrated an improvement in health status in patients with FM in clinical trials, the outcomes are often limited and only benefit a proportion of the subjects. The effectiveness of the different approaches is often short-lived and large variations in treatment response have been cited [8,9]. Many drugs with pharmacologically different mechanisms have demonstrated effectiveness in subgroups of FM patients, but no single drug has addressed the complete range of FM symptoms nor met the needs of all patients consistent with a heterogeneity of the patient population $[9,10]$. Drug treatment remains largely empiric with the condition being managed through targeting individual symptoms where results are highly variable and long-term prospective observational studies have found that outcomes are typically poor. Consequently, current medications are either insufficient to control the symptoms of many patients or difficult to tolerate often due to an apparent high sensitivity to adverse effects of drugs. A high rate of discontinuation $(33-42 \%)$ has been observed in FM phase 3 clinical trials [1]. Thus, the majority of patients with FM only obtain modest relief to drug treatments and general compliance is low.

A number of drugs are currently in development for FM reflecting the interest by the pharmaceutical industry into this condition $[9,10]$. For the majority of drugs that have been or are currently in clinical trials for FM, however, this condition (and thereby the patient population) is secondary to that for which the compound has been initially developed or approved. The lack of understanding of the pathophysiology of FM has prevented the availability of clues for the rational design of mechanistically based drug treatment approaches. As a consequence, attempts to identify new therapies involve either the assessment of existing therapeutic strategies with the potential of improving tolerability, or evaluation of drugs that have previously demonstrated efficacy in another condition for a symptom associated with FM $[1,9]$. The successes of many of the clinical trials have been limited by the complexities of FM. The identification of viable specific drug targets has been hindered by the limited understanding of the pathophysiology of FM and previous difficulty in achieving good drug efficacy in such a heterogeneous patient population.

Many of the clinical trials of potential drug treatments, however, have common limitations and may be criticized for evaluating a population that may not be representative of the general FM population which influence the outcomes. The chronic nature of FM and large intrinsic variability of symptoms, particularly pain, over short periods of time requires pharmacological therapies with long-term efficacy which may not be observed across all symptoms within the first few weeks of treatment, consequently short-term trials (eg up to 12 weeks) will not provide clinically applicable outcomes [1,9-11]. Although evidence from clinical studies indicates that certain pharmacological agents, eg pregabalin and duloxetine, can provide meaningful benefit in FM within one to two weeks with early improvement being a predictor of treatment outcome, proof of adequate long-term efficacy requires attention [12]. Durability of benefit has often been in restricted cohorts (through selective inclusion criteria; eg gender), involving extension studies that have been defined by the restricted criteria of the initial trial. Many clinical trials involved the use of relatively small patient numbers and as a consequence the outcomes are complicated by the heterogeneity of the patient population and proposals that there could be subgroups of patients with FM. An increase in the duration (12 months and greater) and cohort size of trials will provide a more realistic representation of the clinical setting and should not be solely to improve statistical significance for perhaps a weak therapy [13].

The exclusion of patients with other pain conditions and the use of certain analgesics or other medications is common practice to avoid confounding the interpretation of data as well as reporting of adverse effects. Co-morbidities, such as osteoarthritis or rheumatoid arthritis, depression, irritable bowel syndrome, however can be the norm rather than the exception with FM in clinical practice, but patients with co-morbidities are often excluded from clinical trials. This focus to simplify studies, perhaps because of the limited understanding of FM, rather than the requirement of the condition will understandably dictate the outcomes. Again, this is further confounded

*Corresponding author: Kim Lawson, Biomedical Research Centre, Sheffield Hallam University, Faculty of Health and Wellbeing, City Campus, Sheffield, S1 1WB, UK, Tel: +44 (0)114 2253057; E-mail: K.Lawson@shu.ac.uk

Received November 10, 2014; Accepted November 12, 2014; Published November 15, 2014

Citation: Lawson K (2014) Clinical Trials for Patients with Fibromyalgia Syndrome. Clin Exp Pharmacol 4: e134. doi:10.4172/2161-1459.1000e134

Copyright: ( 2014 Lawson K. This is an open-access article distributed under the terms of the Creative Commons Attribution License, which permits unrestricted use, distribution, and reproduction in any medium, provided the original author and source are credited. 
by studies evaluating populations (predominantly female, middle aged, Caucasian, overweight and with symptoms for approximately 10 years) not necessarily representing clinical practice [14].

Pain management has often been a single or the primary symptom endpoint of trials in FM with limited consideration of other symptoms $[1,8,9]$, however a more frequent involvement of quality of life assessment is being recognised. Although the clinician-investigators' identified core domains as most important for assessment in FM studies has often been primarily focused on reducing pain, an OMERACT (Outcome Measures in Rheumatology) Delphi exercise identified several other important patient-reported concerns (e.g. fatigue, sleep disturbance, stiffness, poor concentration and disorganised thinking) [15]. The practicality or availability of assessment of the domains rather than relevance to the condition may often influence the incorporation within clinical trial protocols. Thus directing treatment strategies toward a broad variety of FM symptoms is suggested by these patientreported concerns. The range of FM symptoms and their severity, and the presence of co-morbidities need to be taken in to consideration where the management of patients requires an individualized treatment approach.

Monotherapy randomized placebo-controlled clinical trials in FM have only allowed identification of marginal improvements in the clinical state. Investigation of a single therapy approach in the design of FM clinical trials is not representative of clinical practice where pharmacological and non-pharmacological treatments are incorporated as combination therapies $[1,5]$. The development of flexible or adaptive design would enable trials to be more representative for the patient population with this complex condition (i.e. several fluctuating symptom domains, various co-morbidities) with the necessity of multitherapy approaches [13]. The success of such therapies will give clues to the biology associated with FM and this will lead to the development of more robust and universally accepted diagnostic tools. Improved diagnosis in combination with the development of condition, rather than symptom-focused, therapies will lead to further advancement in clinical trial design.

Therefore there is a need for more naturalistic larger studies, which include patients with various medical co-morbidities, involve concurrent therapies, to address the chronic nature of the condition and to achieve better informed decisions between alternative treatments.

\section{References}

1. Lawson $\mathrm{K}(2008)$ Treatment options and patient perspectives in the management of fibromyalgia: future trends. Neuropsych Dis Treat 4: 1059-1071.

2. Okifuji A, Hare, BD (2013) Management of fibromyalgia syndrome: review of evidence. Pain Ther 2: 87-104.

3. Wolfe F, Smythe HA, Yunus MB, Bennett RM, Bombardier C, et al. (1990) The American College of Rheumatology (1990) criteria for the classification of fibromyalgia. Report of the multicenter criteria committee. Arthritis Rheum 33: 160-172.

4. Jorge LL, Amaro E Jr (2012) Brain imaging in fibromyalgia. Curr Pain Headache Rep 16: 388-398.

5. Goldenberg DL, Bradley LA, Arnold LM, Glass J, Clauw D (2008) Understanding fibromyalgia and its related disorders. Prim Care Companion J Clin Psychiatry 10: $133-144$.

6. Wilson HD, Starz TW, Robinson JP, Turk DC (2009) Heterogeneity within the fibromyalgia population: theoretical implications of variable tender point severity ratings. J Rheumatol 36: 2795-2801.

7. Katz RS, Wolfe F, Michaud K (2006) Fibromyalgia diagnosis: a comparison of clinical, survey, and American College of Rheumatology criteria. Arthritis Rheum 54: 169-176.

8. Glombiewski JA, Sawyer AT, Gutermann J, Koenig K, Rief W, et al. (2010) Psychological treatments for fibromyalgia: a meta-analysis. Pain 151: 280-295.

9. Häuser W, Walitt B, Fitzcharles MA, Sommer C (2014) Review of pharmacological therapies in fibromyalgia syndrome. Arthritis Res Ther 16: 201

10. Mease PJ, Dundon K, Sarzi-Puttini P (2011) Pharmacotherapy of fibromyalgia. Best Pract Res Clin Rheumatol 25: 285-297.

11. Ablin JN, Buskila D (2013) Fibromyalgia syndrome - Novel therapeutic targets Maturitas 75: 335-340.

12. Wang F, Ruberg SJ, Gaynor PJ, Heinloth AN, Arnold LM (2011) Early improvement in pain predicts pain response at endpoint in patients with fibromyalgia. J Pain 12: 1088-1094.

13. Greenhalgh T, Howick J (2014) Evidence based medicine: a movement in crisis? BMJ 348: g3725.

14. Bennett RM, Jones J, Turk DC, Russell IJ, Matallana L (2007) An internet survey of 2,596 people with fibromyalgia. BMC Musculoskelet Disord 8: 27.

15. Mease PJ, Arnold LM, Crofford LJ, Williams DA, Russell IJ, et al. (2008) Identifying the clinical domains of fibromyalgia: contributions from clinician and patient Delphi exercises. Arthritis Rheum 59: 952-960. 